Claremont Colleges

Scholarship@ Claremont

All HMC Faculty Publications and Research

HMC Faculty Scholarship

$11-9-2005$

\title{
Using Ultrasonic Atomization to Produce an Aerosol of Micron-scale Particles
}

Thomas D. Donnelly

Harvey Mudd College

J. Hogan '03

Harvey Mudd College

A. Mugler'04

Harvey Mudd College

M. Schubmehl '02

Harvey Mudd College

N. Schommer'04

Harvey Mudd College

See next page for additional authors

\section{Recommended Citation}

Using ultrasonic atomization to produce an aerosol of micron-scale particles. T. D. Donnelly, J. Hogan, A. Mugler, M. Schubmehl, N. Schommer, A. J. Bernoff, S. Dasnurkar, and T. Ditmire, Rev. Sci. Instrum. 76, 113301 (2005).

This Article is brought to you for free and open access by the HMC Faculty Scholarship at Scholarship @ Claremont. It has been accepted for inclusion in All HMC Faculty Publications and Research by an authorized administrator of Scholarship @ Claremont. For more information, please contact scholarship@cuc.claremont.edu. 
Authors

Thomas D. Donnelly, J. Hogan '03, A. Mugler '04, M. Schubmehl '02, N. Schommer '04, Andrew J. Bernoff, S. Dasnurkar, and T. Ditmire 


\title{
Using ultrasonic atomization to produce an aerosol of micron-scale particles
}

T. D. Donnelly, ${ }^{\text {a) }}$ J. Hogan, A. Mugler, M. Schubmehl, and N. Schommer

Department of Physics, Harvey Mudd College, Claremont, California 91711

A. J. Bernoff

Department of Mathematics, Harvey Mudd College, Claremont, California 91711

S. Dasnurkar

Department of Electrical and Computer Engineering, University of Texas at Austin, Austin, Texas 78712

T. Ditmire

Department of Physics, University of Texas at Austin, Austin, Texas 78712

(Received 6 May 2005; accepted 2 October 2005; published online 9 November 2005)

\begin{abstract}
A device that uses ultrasonic atomization of a liquid to produce an aerosol of micron-scale droplets is described. This device represents a new approach to producing targets relevant to laser-driven fusion studies, and to rare studies of nonlinear optics in which wavelength-scale targets are irradiated. The device has also made possible tests of fluid dynamics models in a novel phase space. The distribution of droplet sizes produced by the device and the threshold power required for droplet production are shown to follow scaling laws predicted by fluid dynamics. () 2005 American Institute of Physics. [DOI: 10.1063/1.2130336]
\end{abstract}

\section{INTRODUCTION}

Particles larger than atomic clusters yet smaller than macroscopic solids are of interest for a variety of experiments that study laser-matter interactions. For example, such particles will: improve neutron yield when used as laser targets in laser-driven fusion experiments; ${ }^{1-3}$ serve as a testmedia for models of the nonlinear optical phenomena that arise when intense light interacts with a wavelength-scale particle; ${ }^{4-6}$ and be used to test fluid models of droplet formation in a novel phase space. ${ }^{7}$ We describe here an apparatus capable of producing a dense aerosol of micron-scale particles that will be useful in each of these studies.

To date, small scale laser-driven fusion experiments have been carried out by irradiating deuterium clusters with an intense laser pulse. These clusters are typically generated by cooling a high-pressure deuterium gas and then passing it through a small orifice into vacuum. The gas forms clusters as it expands into vacuum, and these clusters have been measured up to sizes of $\sim 100 \AA$ for a deuterium gas cooled to $80 \mathrm{~K}$ at a pressure of $70 \mathrm{~atm}^{1}$ This is roughly the maximum cluster size that can be produced by this method because further cooling is negated by mechanical energy that is added to the system when the gas jet is activated. In these experiments, the neutron yield was measured to increase dramatically with increasing cluster size, rising from $\sim 10^{2}$ neutrons/ shot for $30 \AA$ clusters to $\sim 10^{4}$ neutrons shot for $55 \AA$ clusters. The resultant maximum neutron yield in these experiments is $\sim 10^{5}$ neutrons $/ \mathrm{J}(120 \mathrm{~mJ}$ of input laser energy), which is comparable to the neutron/J yield in laser-driven

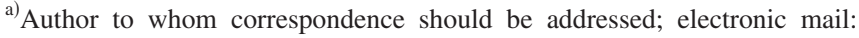
tom_donnelly@hmc.edu
}

fusion studies carried out at Lawrence Livermore National Laboratory on the now decommissioned NOVA laser.

The observed steep dependence of neutron yield on cluster size is predicted by models of a Coulomb-exploding cluster; $^{1}$ if even larger deuterium clusters can be produced, larger neutron yields are expected. Current experiments are run under conditions that produce a deuterium plasma with a temperature of $\sim 10 \mathrm{keV}$, while the deuterium-deuterium fusion cross-section peaks at temperatures near $2 \mathrm{MeV}$. In effect, current experiments only take advantage of the vanishing tail of the fusion cross-section curve, so a large gain in neutron yield can be expected for incremental increases in the deuterium plasma temperature. One way to achieve higher plasma temperatures is by irradiating larger clusters, and the device described here suggests a method for generating such laser targets. Note, however, that for this device the laser target would be heavy water, not pure deuterium.

Beyond improving fusion yields, larger particles will be useful in studies of nonlinear optics. In particular, wavelength-scale particles provide a means for studying nonlinear light-matter interactions in a regime where there is a paucity of experimental and theoretical work, but there exists the promise of Mie-enhanced interactions. Although linear scattering from arbitrary sized spheres has been a solved problem for a long time, it is notable that there is no general theoretical description for nonlinear optical processes that result when a wavelength-scale particle interacts with intense light-not even for the simplest case of second-harmonic generation from a centrosymmetric sphere made of isotropic material. Dadap has developed a theoretical treatment for sphere sizes much smaller than the wavelength, where the Rayleigh-Gans approximation holds, ${ }^{5,6}$ while a paper by Bennemann ${ }^{4}$ has proposed a model which predicts Mie en- 
hancements in the production of low-order harmonics by wavelength-scale particles. In Bennemann's theory the fundamental induced surface charge, as calculated with Mie theory, is likened to an anharmonic oscillator which gives rise to higher modes of oscillation. These higher-order modes of oscillating charge result in high-order harmonic radiation. Each of these models is phenomenological in that the mechanism for creating harmonic radiation is not derived but rather introduced as a polarization constant, or as a simplified harmonic expansion. The need for a general description of these processes is more pressing as Mie enhancements are being exploited to improve the yield of both low- and high-order nonlinear processes. ${ }^{5,8-11}$

Experimental results on the angular dependence of thirdharmonic generation from larger particles (water droplets) with diameter of many wavelengths $(8-32 \mu \mathrm{m})$ have also been published. ${ }^{12}$ These results showed reasonable agreement between their model and the angular distribution of the measured third-harmonic signal, and demonstrate that the study of harmonic generation from particles can yield important information on the field distribution around a particle. The study also suggests that microplasma particles near resonance may be an ideal medium for efficient harmonic generation. For example, the use of atomic clusters as a laser target offers the possibility of generating high harmonics with solid-like efficiencies, but in an extended medium. Preliminary and promising studies of this possibility have been undertaken with nanometer size clusters, ${ }^{13,14}$ however a comprehensive model of harmonic generation from clusters does not exist. Beyond atomic clusters, similar benefits to the harmonic generation process may be expected when using wavelength-scale $(\sim 1 \mu \mathrm{m})$ particles. And, in this case, there may be the additional benefit of an increased yield in the harmonic generation process due to Mie enhancements in the laser-particle interaction.

Previously, we have performed experiments in a more nonlinear regime (laser intensity $\sim 10^{17} \mathrm{~W} / \mathrm{cm}^{2}$ ) using an earlier source of micron-scale droplets. A spray of droplets was produced by forcing a liquid under high pressure through a small orifice. Each spray of the water jet produced a set of droplets ranging in size between 1 and $2 \mu \mathrm{m}$. The droplet source consisted of a gas line backed with highpressure argon (at 67 bar), an inert propellant gas, over a liquid reservoir. The line terminated in a solenoid driven pulsed valve with a $750 \mu \mathrm{m}$ orifice. The valve was pulsed to cause propellant gas carrying liquid droplets to stream through the orifice. Experiments examining hard x-ray production were conducted using these water droplets to gain insight into fast electron production, and showed that the electron and field dynamics are different than in similar intensity interactions with planar solids. ${ }^{8}$

The experiments found that when irradiated with $p$-polarized light the droplets produced hot electron temperatures nearly a factor of 2 times larger than that from solid $\mathrm{CH}$ targets. Particle-in-cell simulations confirmed this observed enhancement in electron temperature. This observed enhancement can be ascribed to a combination of field enhancements around the droplet and to Brunel-type absorption ${ }^{15}$ by electrons whose trajectories undergo numerous surface encounters (trajectories at an infinite plane usually traverse the surface only once).

While our gas jets, as well as others used to produce micron scale droplets, ${ }^{10,11,16,17}$ have found use in these sorts of experiments, they are limited. It is difficult to predict the size of droplets that are produced by a jet under a given set of experimental parameters (orifice size, backing pressure, orifice shape, etc.), and ultimately one must rely on numeric hydrodynamic simulations to design jets for a specific droplet size. ${ }^{18}$ Moreover, the distribution of droplets produced by these jets can have significant spatial inhomogeneities, and, importantly, the distribution of droplet sizes produced by the jet is not reported. In practice, gas jets are often used empirically, varying experimental parameters and then measuring the average size of the resulting droplets to see if an appropriate experimental phase space has been found. The ultrasonic atomization approach to target production overcomes many of the difficulties inherent in the gas jet.

In many experiments of interest it is important to be able to deliver a dense aerosol of particles to the laser focus, e.g., in laser-fusion experiments. The gas jet method of particle production is capable of doing this, producing sprays with average atomic densities near $10^{19}$ atoms $/ \mathrm{cm}^{3} .{ }^{11}$ As will be discussed in the following, the aerosol produced using ultrasonic atomization is comparable in density to that produced by a gas jet.

Finally, fluid dynamics is a field in which our droplet source has already found use and promises more utility. The theory of Faraday excitation can describe droplets formed through ultrasonic atomization of a driven, viscous, incompressible fluid. ${ }^{19-23}$ Models show that the size of droplets produced by harmonic excitation of the fluid surface is related to the driving frequency and properties of the fluid. The relationship between droplet size and these parameters breaks down into two regimes of phase-space, the inviscid (low driving frequency, surface tension dominated) and viscous (high driving frequency, viscosity dominated). Fluid models of droplet formation through Faraday excitation have been tested in the low driving frequency limit by various authors, ${ }^{24}$ and have been shown to hold true in this limit. We have used the predictions of these models to guide the design of our droplet source, and, in turn, used the source to test the models at driving frequencies which are orders of magnitude higher than previously investigated. ${ }^{7}$ These tests of droplet size predictions took place in the inviscid regime of droplet formation, and here we report on critical power measurements that occur in the viscous regime. The next iteration of the droplet source will allow tests of droplet size predictions in the viscous regime.

\section{BASIC THEORY}

\section{A. Droplet diameter}

Scaling laws, or a more sophisticated linear stability analysis, can be used to establish how a fluid's properties (kinematic viscosity, $\nu$; surface tension, $\sigma$; and density, $\rho$ ) and the frequency, $\omega$, at which that fluid is driven are related 
to the diameter, $d$, of the droplets that are generated at the fluid's surface. ${ }^{7}$ The relations appear in two regions of parameter space,

$$
\begin{aligned}
& d=2 \pi c_{1}\left(\frac{\sigma}{\rho}\right)^{1 / 3}\left(\frac{2}{\omega}\right)^{2 / 3} \quad \text { (inviscid), } \\
& d=2 \pi c_{2}\left(\frac{9 \nu}{2 \omega}\right)^{1 / 2} \quad \text { (viscous), }
\end{aligned}
$$

where $c_{i}$ is a constant that can be found analytically in the inviscid regime, or numerically in the viscous regime. We have measured the value $c_{1}=0.35 \pm 0.03$ in the inviscid regime, consistent with calculations. The crossover point between the inviscid and viscous regimes occurs at a dimensionless frequency, $\Omega$ equal to 0.1756 , where the dimensionless frequency is defined as

$$
\Omega=\omega\left(\frac{\nu^{3} \rho^{2}}{\sigma^{2}}\right) .
$$

The system is in the viscous regime if $\Omega \gg 0.1756$, and the inviscid regime if $\Omega \ll 0.1756$.

From Eqs. (1)-(3) one can determine how to make droplets of a desired size: For a given sample, fluid properties are measured and then the sample is driven at an appropriate frequency (viscous or inviscid regime) to produce a droplet of the specified size. For example, at room temperature, water has fluid property values of $\sigma=0.073 \mathrm{~N} / \mathrm{m}, \rho$ $=997 \mathrm{~kg} / \mathrm{m}^{3}, \nu=1.0 \times 10^{-6} \mathrm{~m}^{2} / \mathrm{s}$. To make a droplet with $d=1 \mu \mathrm{m}$, therefore, the fluid must be driven at frequency $f=\omega / 2 \pi=8.5 \mathrm{MHz}$. This is the inviscid regime, since, in this case, $\Omega=0.01 \ll 0.1756$.

Note that here, as compared to the case of gas jets, there is no guess-work about the size of the particle that is produced, and the theory is straightforward to implement. All that is required to produce droplets of a desired size is knowledge of the fluid parameters, and the ability to drive the fluid at a given frequency.

In analogy with the dimensionless frequency, it is often convenient to relate the physical diameter of the droplet to a so-called dimensionless diameter, $D$,

$$
D=d\left(\frac{\sigma}{\rho \nu^{2}}\right) .
$$

This formulation will be particularly useful when comparisons are made between experimental data and the fluid theory predictions which describe droplet formation by Faraday excitation.

\section{B. Input power requirement}

When driving a fluid, there is some acceleration of the fluid above which droplets begin to pinch off from the surface. This is called the critical acceleration $a_{c}$. Using dependence arguments and dimensional analysis, it can be shown that

$$
a_{c}=b_{1}\left(\frac{\sigma}{\rho}\right)^{1 / 3} \omega^{4 / 3} \quad \text { (inviscid) }
$$

$$
a_{c}=b_{2} \nu^{1 / 2} \omega^{3 / 2} \quad \text { (viscous), }
$$

where $b_{1}$ and $b_{2}$ are constants of proportionality. These constants have been measured experimentally by Goodridge et al. and are found to be $b_{1}=0.261$ and $b_{2}=1.306$. ${ }^{23}$ Because the physics which determines the critical acceleration-the minimum acceleration at which droplets are ejected from a driven surface-is different from that which determines the droplet's size once it is ejected, these two phenomena cross between the inviscid and viscous regime at different dimensionless frequencies. The crossover frequency for the diameter regimes is almost four orders of magnitude greater than that for the acceleration regimes. As mentioned previously, when determining droplet size, the crossover between the regimes occurs at $\Omega=0.1756$, while for the critical acceleration the crossover occurs at a dimensionless frequency $\Omega$ $=6.37 \times 10^{-5}$. This difference in crossovers is physically reasonable; it simply suggests that viscous effects dominate the amplitude of the surface wave, which determines the critical ejection point, at a lower frequency than they do the wavelength, which governs the droplet size.

The critical power $P_{c}$-the minimum power the oscillator needs to input to the fluid to achieve atomization — can be determined from $a_{c}$. Noting that $P=F v=m a v$, where $m$ is the mass of the fluid column being driven and $F$ is the force applied to the column directly above the oscillator, and assuming that the fluid is driven sinusoidally in the viscous regime [Eq. (6)], one finds

$$
P_{c} \sim \frac{m a_{c}^{2}}{\omega} \sim m \nu \omega^{2} .
$$

While Eqs. (1) and (2) show that smaller droplets can be created by driving a sample fluid at higher frequencies, Eq. (7) indicates that going to ever-higher driving frequencies to achieve smaller droplets-droplets that will be useful in studies of fusion and nonlinear optics-is an energetically costly approach. To determine whether or not this approach is, in practice, a feasible way to proceed toward making smaller and smaller droplets, we make critical power measurements for a number of fluids and, using Eq. (7), extrapolate to determine the power requirements for a high frequency driving system capable of producing droplets that are much smaller than $1 \mu \mathrm{m}$.

\section{Temperature dependence of fluid properties}

Fluid parameters are temperature dependent, hence it is necessary to know how they vary as a function of temperature for each fluid of interest. The temperature dependence makes it necessary to record fluid temperature while making any measurements, so that data and theory can be properly compared. Our device is characterized using water and water-glycerine solutions, where the water-glycerine solutions are specified by indicating the percentage of glycerine by weight in the solution. Tables I-III provide values for polynomial fits of the fluid parameters, as a function of temperature, for water, $20 \%$ glycerine, $40 \%$ glycerine, $60 \%$ glycerine and ethanol. The parameter values found from these tables are used to calculate $d, \Omega$, and $D$, or any other 
TABLE I. Fluid density polynomial interpolations. The interpolation is of the form $\rho(T)=A_{0}+A_{1} T+A_{2} T^{2}$ $+A_{3} T^{3}+A_{4} T^{4}+A_{5} T^{5}$. The temperature $T$ ranges from 0 to 100 in degrees Celsius. The polynomial gives the density in units of $\mathrm{kg} / \mathrm{m}^{3}$.

\begin{tabular}{lcccccc}
\hline \hline \multicolumn{1}{c}{ Density } & $A_{0}$ & $A_{1}$ & $A_{2}$ & $A_{3}$ & $A_{4}$ & $A_{5}$ \\
\hline Water & 999.842 & 0.04935 & $-7.44 \times 10^{-3}$ & $4.048 \times 10^{-5}$ & $-1.26 \times 10^{-7}$ & 0 \\
$20 \%$ glycerine & 1051.61 & -0.1777 & $-2.85 \times 10^{-3}$ & $-9.711 \times 10^{-6}$ & $8.36 \times 10^{-8}$ & 0 \\
$40 \%$ glycerine & 1106.6 & -0.2596 & $-5.74 \times 10^{-3}$ & $4.506 \times 10^{-5}$ & $-1.61 \times 10^{-7}$ & 0 \\
$60 \%$ glycerine & 1163.5 & -0.4134 & $-4.2 \times 10^{-3}$ & $4.1096 \times 10^{-5}$ & $-1.73 \times 10^{-7}$ & 0 \\
Ethanol & 806.50 & -0.8702 & $1.37 \times 10^{-3}$ & $-2.60 \times 10^{-5}$ & $-3.09 \times 10^{-8}$ & $1.2 \times 10^{-9}$ \\
\hline \hline
\end{tabular}

value of interest, corrected for temperature. The tables provide each of the fluid parameters using a polynomial interpolation of the temperature dependence.

The density interpolation is given by

$$
\rho(T)=A_{0}+A_{1} T+A_{2} T^{2}+A_{3} T^{3}+A_{4} T^{4}+A_{5} T^{5},
$$

where the coefficients $A_{i}$ depend on the fluid and are given by Table I. The surface tension interpolation is

$$
\sigma(T)=B_{0}+B_{1} T+B_{2} T^{2}+B_{3} T^{3}+B_{4} T^{4}+B_{5} T^{5},
$$

where the coefficients $B_{i}$ are shown in Table II. Finally, the viscosity interpolation is

$$
\eta(T)=C_{0}+C_{1} T+C_{2} T^{2}+C_{3} T^{3}+C_{4} T^{4}+C_{5} T^{5},
$$

where the coefficients $C_{i}$ are shown in Table III. For each of these three interpolation functions the tabulated coefficients assume that the temperature is in units of degrees Celsius and ranges from 0 to $100{ }^{\circ} \mathrm{C}$. Note that Table III and Eq. (11) give the absolute viscosity, $\eta$, not the kinematic viscosity, $\nu$. The kinematic viscosity is found from the ratio of the viscosity and the density, $\nu=\eta / \rho$, at a given temperature.

Over the course of a droplet sizing measurement, the temperature of the fluid usually fluctuated within about $1{ }^{\circ} \mathrm{C}$. Since these fluctuations are small, the average measured temperature of the fluid is used in Eqs. (8)-(10) to calculate $\rho, \sigma$, and $\nu$. However, the average temperature often differed significantly between data sets, and the effect that this has on each of the fluid properties cannot be ignored when calculating the nondimensional droplet diameter $D$ and driving frequency $\Omega$. In addition, the small temperature uncertainty $\Delta T$ observed during each individual data set was used to calculate the uncertainties for $\rho, \sigma$, and $\nu$. These uncertainties contribute to the reported uncertainty in both $D$ and $\Omega$.

\section{APPARATUS}

Although ultrasonic atomizers, also called nebulizers, are commercially available at a few frequencies, any attempt to study a range of frequencies requires a custom-built solution. It is known that the oscillation frequency of a device is determined by the piezoelectric ceramic disc that actually performs the atomization-two discs of the same geometry that are made of the same material will differ in frequency only if they differ in thickness, with the thinner disc operating at the higher frequency. This makes it fairly straightforward to modify a commercial atomizer to operate at a different frequency by swapping the manufacturer's piezo for a custom-made one of a different thickness.

The disc operates by deforming when a voltage is placed across it, so the key to using it for atomization is to supply enough power at the proper frequency so that the piezo"s vibrations excite the surface of the water. It is a characteristic property of the ceramic piezos that they have a narrow resonance peak, so that they can essentially only be excited at a single frequency or some harmonic thereof. In practice, the piezo responds only to the dominant frequency of the driving voltage, and therefore even somewhat messy driving voltage wave forms can be used to efficiently drive the piezo. This resonant behavior helps make the atomizing device robust. The collection of piezoelectric discs used in this experiment were all the same material, crystal number 841 manufactured by American Piezo. ${ }^{25}$ All the piezos had the same diameter of $19.5 \mathrm{~mm}$, so the resonance frequency of each disc was controlled by its thickness alone. The resonant frequency of the piezo is $f=N_{T} / h$, where $f$ is the resonant frequency, $h$ is the thickness of the piezo, and $N_{T}$ is a constant with a value of $2005 \mathrm{~m} / \mathrm{s}$ for our material.

Ideally, for the studies of interest here, the piezo would be driven using a high-power sine-wave generator capable of

TABLE II. Fluid surface tension polynomial interpolations. The interpolation is of the form $\sigma(T)=B_{0}+B_{1} T$ $+B_{2} T^{2}+B_{3} T^{3}+B_{4} T^{4}+B_{5} T^{5}$. The temperature $T$ ranges from 0 to 100 in degrees Celsius. The polynomial gives the surface tension in units of $\mathrm{N} / \mathrm{m}$.

\begin{tabular}{llllccc}
\hline \hline \multirow{2}{*}{ Surface tension } & \multicolumn{1}{c}{$B_{0}$} & \multicolumn{1}{c}{$B_{1}$} & \multicolumn{1}{c}{$B_{2}$} & \multicolumn{1}{c}{$B_{3}$} & $B_{4}$ & $B_{5}$ \\
\hline Water & 0.07565 & $-1.39652 \times 10^{-4}$ & $-2.9969 \times 10^{-7}$ & $2.2616 \times 10^{-10}$ & 0 & 0 \\
$20 \%$ glycerine & 0.07468 & $-2.4589 \times 10^{-4}$ & $3.69 \times 10^{-6}$ & $-5.02 \times 10^{-8}$ & $2.2 \times 10^{-10}$ & 0 \\
$40 \%$ glycerine & 0.0721 & $-1.32 \times 10^{-4}$ & $6.06 \times 10^{-7}$ & $-1.19 \times 10^{-8}$ & $5.6 \times 10^{-11}$ & 0 \\
$60 \%$ glycerine & 0.06958 & $-1.024 \times 10^{-4}$ & $4.255 \times 10^{-7}$ & $-8.655 \times 10^{-9}$ & $3.7 \times 10^{-11}$ & 0 \\
Ethanol & 0.0241 & $-9.07833 \times 10^{-5}$ & $3.15 \times 10^{-7}$ & $-3.7 \times 10^{-9}$ & 0 & 0 \\
\hline \hline
\end{tabular}


TABLE III. Fluid viscosity polynomial interpolations. Note that this interpolation does not give the kinematic viscosity, $\nu$, but the viscosity, $\eta$. Kinematic viscosity is found by dividing the viscosity by the density, at a given temperature, $\nu=\eta / \rho$. The interpolation is of the form $\eta(T)=C_{0}+C_{1} T+C_{2} T^{2}+C_{3} T^{3}+C_{4} T^{4}+C_{5} T^{5}$. The temperature $T$ ranges from 0 to 100 in degrees Celsius. The polynomial gives the viscosity in units of $\mathrm{kg} / \mathrm{m} / \mathrm{s}$.

\begin{tabular}{lcccccc}
\hline \hline \multicolumn{1}{c}{ Viscosity } & $C_{0}$ & \multicolumn{1}{c}{$C_{1}$} & $C_{2}$ & $C_{3}$ & $C_{4}$ & $C_{5}$ \\
\hline Water & 0.00179 & $-5.892 \times 10^{-5}$ & $1.28 \times 10^{-6}$ & $-1.754 \times 10^{-8}$ & $1.30 \times 10^{-10}$ & $-3.95 \times 10^{-13}$ \\
20\% glycerine & 0.00344 & $-1.28 \times 10^{-4}$ & $2.87 \times 10^{-6}$ & $-3.797 \times 10^{-8}$ & $2.57 \times 10^{-10}$ & $-6.25 \times 10^{-13}$ \\
$40 \%$ glycerine & 0.00824 & $-3.639 \times 10^{-4}$ & $9.12 \times 10^{-6}$ & $-1.32 \times 10^{-7}$ & $1.0 \times 10^{-9}$ & $-3.05 \times 10^{-12}$ \\
$60 \%$ glycerine & 0.02987 & $-1.62 \times 10^{-3}$ & $4.51 \times 10^{-5}$ & $-6.85 \times 10^{-7}$ & $5.3 \times 10^{-9}$ & $-1.63 \times 10^{-11}$ \\
Ethanol & 0.00177 & $-3.63 \times 10^{-5}$ & $4.23 \times 10^{-7}$ & $-3.05 \times 10^{-9}$ & $1.06 \times 10^{-11}$ & 0 \\
\hline \hline
\end{tabular}

frequencies in the $1-50 \mathrm{MHz}$ range. In practice, such a power supply is not readily available to us, so the piezos are driven using commercially available driver boards from American Piezo that have been modified. A circuit diagram of the boards is shown in Fig. 1. The circuit board takes a dc signal and converts it into ac at a frequency determined primarily by the piezo, and the three capacitors located in the center of the board. The basic circuit can be modified so that it produces desired frequencies, as described in the caption of Fig. 1.

Driving a piezo with one of the boards is as simple as mounting the piezo on the bottom of a container of water, connecting the wires to a power supply, and then slowly increasing the supply voltage until atomization is observed, or until the voltage reaches the board"s maximum of $40 \mathrm{~V}$ $(80 \mathrm{~V}$ peak-peak). A plastic container with a hole in the bottom has been mounted to one of the piezo holders using waterproof aquarium sealant. This makes it fairly easy to contain the aerosol that is generated and direct it into the sizing apparatus as needed. Two small holes allow tubes to be connected to the closed container: one from a bottle of inert gas to create a slight overpressure, and one to a hose leading to a small orifice that empties into the scattering apparatus. The tube leading to the jet has a drainage tube attached at its midpoint, to allow condensation to drain out of the tube. Without this attachment, condensation can quickly block the hose. Cooling fans were used to increase the lifetime of the driver boards, and when a board was driven at high power (above approximately $50 \mathrm{~V}$ peak-peak) copper tube carrying chilled water was attached to the metal heat sinks. A small fountain pump and basin of ice water were used to circulate ice water through these tubes.

To characterize the droplets produced by our source we have measured the size-distribution of droplets, the density of the droplets, and the minimum powered required to produce the droplets. Some of the details provided here for the size-distribution and aerosol density measurements also appear in a previous publication. ${ }^{7}$ For the droplet sizing measurements, piezoelectric oscillators with resonant frequencies ranging from 1.65 to $2.42 \mathrm{MHz}$ were used to create the droplets. These piezoelectrics and driving boards were purchased from American Piezo (part 50-1010 for the $1.65 \mathrm{MHz}$ board, and $50-1025$ for the $2.42 \mathrm{MHz}$ board). The actual driving frequency of the board is established by the piezo being driven-in effect, the sharp resonance of the piezo dominates the broader resonance of the driving board. The driving fre- quency of the system was thus controlled by varying the thickness of the piezo placed in the driving circuit. Various water-glycerine solutions were used as test fluids in order to vary the sample density, surface tension, and kinematic viscosity, with concentrations of glycerine ranging from $0 \%$ to $60 \%$ by weight. (Solutions with glycerine concentrations above $60 \%$ were too viscous to be atomized by our system.) This combination of fluid parameters and driving frequencies has allowed data to be obtained over more than two decades of nondimensional driving frequency.

\section{EXPERIMENTS}

Droplets were sized using Mie scattering techniques. As shown in Fig. 2, a $1 \mathrm{~W}$ argon-ion laser operating at $488 \mathrm{~nm}$ was scattered by the aerosol. The angular scattering pattern thus produced is characteristic of the ratio of the droplet size to the incident wavelength. This ratio is called the size parameter, and it is defined as

$$
x=2 \pi r / \lambda,
$$

where $r$ is the droplet radius and $\lambda$ is the wavelength of the incident light. For each aerosol produced, the angular scattering pattern was measured and then Mie theory was used to determine a droplet size.

To measure the angular scattering pattern, the aerosol was directed over the center of a rotation stage. An amplified photodetector ${ }^{26}$ was connected to the rotation stage via a rail. The iris on the rail between the center of the rotation stage and the photodetector restricted $\theta$ in Fig. 2 to angles larger

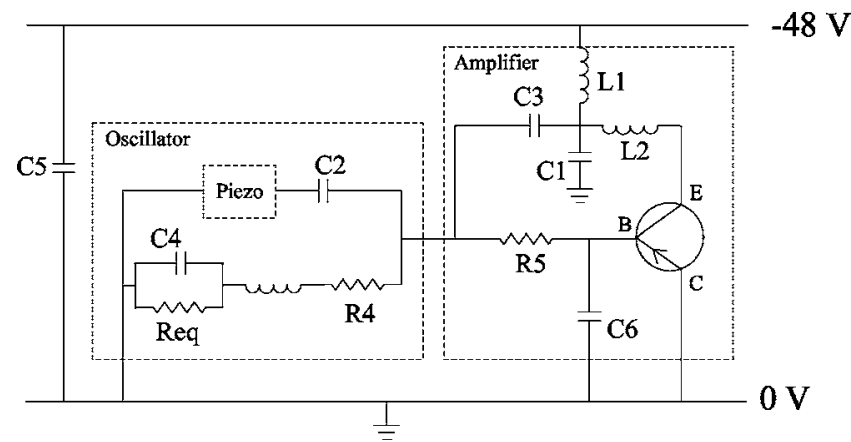

FIG. 1. A circuit diagram of the driver board used to drive the piezos. To make the board drive voltage at various frequencies, the following component values are useful: $1 \mathrm{MHz}, C_{2}=C_{3}=72 \mathrm{nF}, C_{1}=2.2 \mathrm{nF} ; 1.65 \mathrm{MHz}, C_{2}$ $=C_{3}=47 \mathrm{nF}, C_{1}=1.5 \mathrm{nF} ; 2.42 \mathrm{MHz}, C_{2}=C_{3}=33 \mathrm{nF}, C_{1}=1.2 \mathrm{nF} ; 3.3 \mathrm{MHz}$, $C_{2}=C_{3}=22 \mathrm{nF}, C_{1}=0.72 \mathrm{nF} ; 5 \mathrm{MHz}, C_{2}=C_{3}=15 \mathrm{nF}, C_{1}=0.56 \mathrm{nF}$. 


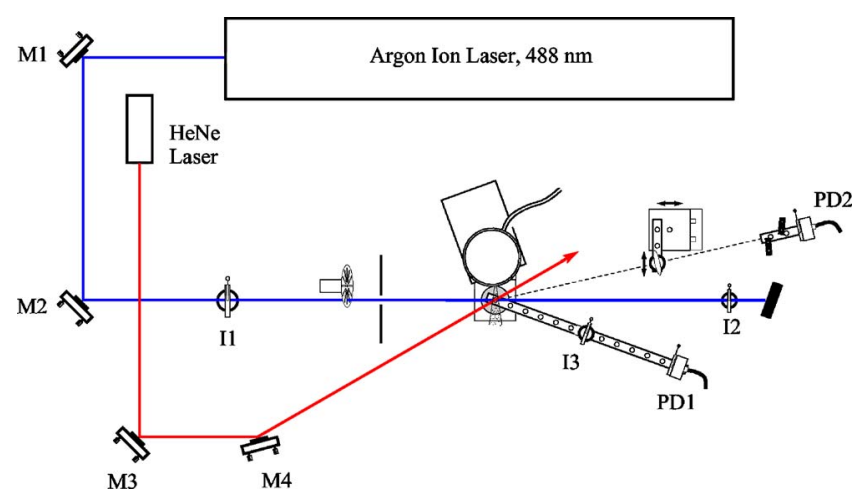

FIG. 2. The experimental setup used for measuring droplet sizes. Droplets were generated by ultrasonic atomization using a rapidly driven piezo and were sized using Mie scattering techniques. A $1 \mathrm{~W}$ argon-ion laser operating at $488 \mathrm{~nm}$ was used for the incident beam. To correct for variations in aerosol density and laser intensity a reference arm (PD2) is used to normalize the intensity measured at each angle $\theta$ (PD1). A helium-neon laser is used for alignment purposes. At the point where the argon-ion and heliumneon beams cross, the aerosol is blown out of the chamber in which it is created. A chopper and lock-in amplifier are used for enhanced signal detection. $\mathrm{M}$ refers to a mirror; I refers to an iris; and PD refers to a photodetector.

than $5^{\circ}$. The scattered light intensity was measured at 15 different angles between $6^{\circ}$ and $30^{\circ}$ with a resolution of $0.5^{\circ}$.

During the course of a single scattering measurement, the density of the aerosol can fluctuate for two reasons. First, a compressed-air source is used to slightly over pressure the chamber in which the aerosol is created, thus forcing the aerosol out of the chamber through an orifice. The effect of the orifice is to localize the aerosol as it exits the chamber and interacts with the laser. Because the compressed-air source pressure is somewhat variable, so is the aerosol density. Second, the aerosol density can fluctuate because droplets are ejected from the fluid surface in an erratic fashion; this is inherent in the droplet ejection mechanism. In order to correct for such variations in aerosol density, the signal from a reference photodetector was used to normalize the detector signal. The light scattered by the aerosol column was imaged onto the reference photodetector. By imaging the entire illuminated column onto the detector, an average is taken over all local variations in intensity. Normalization by this method has the added advantage of automatically correcting for any variations in incident laser intensity that might occur during a data set.

The incident laser beam was chopped, and analog lock-in amplifiers were used to enhance signal detection. The lock-in amplifiers were especially important for experiments involving high concentrations of glycerine. Generally, the higher the percent concentration of glycerine, the more difficult the fluid is to atomize, and so for high concentrations the amount of aerosol generated can be small.

Typical angular scattering data are shown in Fig. 3(a). One sample curve for each of the measured fluids is shown, where the fluids were driven at either 2.14 or $2.42 \mathrm{MHz}$. The scattering pattern for $60 \%$ glycerine is notably flatter than the other fluids, and this is because the $60 \%$ glycerine is driven very hard to produce atomization. This means that the instability associated with the surface mode of oscillation is
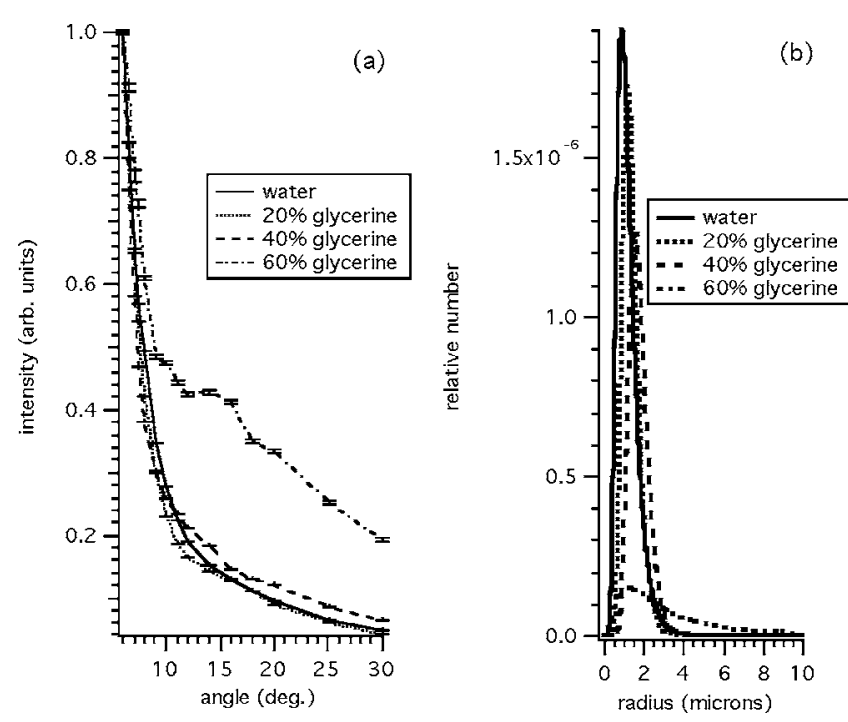

FIG. 3. A sample of angular scattering patterns and the inferred droplet size distributions. (a) The angular scattering measurements, for a variety of fluids, made using the experimental setup shown in Fig. 2. Computer models of Mie scattering are used to infer the lognormal distributions shown in (b). The data were taken at driving frequencies of $2.42 \mathrm{MHz}$ (water), $2.42 \mathrm{MHz}$ (20\% glycerine), $2.15 \mathrm{MHz}$ (40\% glycerine), and $2.15 \mathrm{MHz}$ (60\% glycerine).

present over a broader frequency range and therefore a more disperse size distribution of droplets is created.

Once the angular scattering pattern was measured, Mie scattering theory was used to infer a droplet size. ${ }^{27}$ Data sets such as the one shown in Fig. 3(a) were numerically fit with the help of a modified version of the FORTRAN MIEV0 program. ${ }^{28}$ The aerosols produced in this experiment are not monodisperse because of the complicated process of droplet ejection, and other processes such as droplet cohesion. Because of these effects an aerosol must be described by a distribution of droplet sizes. Following Kerker, we model the aerosols using a lognormal size distribution. ${ }^{29}$ This distribution is given by

$$
n(r)=\frac{1}{\sqrt{2 \pi} s r} \exp \left(\frac{-(\ln (r)-\ln (R))^{2}}{2 s^{2}}\right),
$$

where $n(r)$ is the relative abundance of droplets with radius $r, \ln (R)$ is the mean value of $\ln (r)$, and $s$ characterizes the variance of the distribution. Averaged over all samples, we find that the average ratio of the distribution's width to the peak droplet size is 0.67 . Figure 3(b) shows the lognormal fits that correspond to the angular scattering patterns in Fig. 3(a). Once the lognormal distribution has been determined, droplet size is recorded as the peak radius of the lognormal distribution. In performing an accurate fit it is important to know the index of refraction of the droplet medium; we calculate the index of refraction of our water-glycerine mixtures by taking an average, weighted by mass, of the water and glycerine indecies.

Figure 4 shows a typical series of measurements, taken over a single day, for water droplets created at a driving frequency of $1.52 \mathrm{MHz}$. Figure 4(a) shows the angular scattering patterns and Fig. 4(b) shows the inferred lognormal 

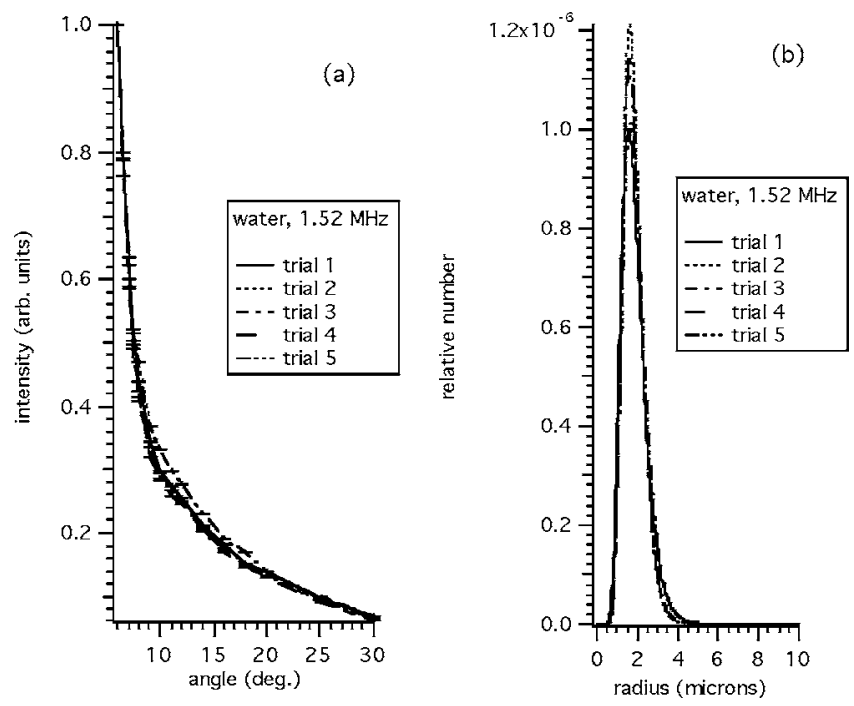

FIG. 4. Angular scattering patterns and the inferred lognormal distributions for water driven at $1.52 \mathrm{MHz}$. The results of five data sets, taken over many hours, are displayed in order to demonstrate the repeatability of the droplet source. The results of the lognormal fits are: trial $1, r_{\text {peak }}=1.56 \mu \mathrm{m}$, $s=0.47 \mu \mathrm{m}$; trial $2, r_{\text {peak }}=1.61 \mu \mathrm{m}, s=0.39 \mu \mathrm{m}$; trial $3, r_{\text {peak }}=1.59 \mu \mathrm{m}$, $s=0.41 \mu \mathrm{m}$; trial $4, r_{\text {peak }}=1.62 \mu \mathrm{m}, s=0.39 \mu \mathrm{m}$; trial $5, r_{\text {peak }}=1.66 \mu \mathrm{m}$, $s=0.43 \mu \mathrm{m}$.

distributions. The data demonstrate repeatability and agreement with the theory encapsulated in Eqs. (1)-(3).

In addition to knowing the distribution of droplet sizes within the aerosol, it is convenient to know the aerosol density. Once the droplet sizes are know, then, experimentally, the problem of measuring aerosol density can be reduced to a measurement of light transmission along the beam line. ${ }^{29}$ The transmission measurement was made by switching the aerosol source on and off. As shown in Fig. 5, a glass slide was used to pick off a small fraction of the light from the beam just after it passed through the aerosol. Then, the aerosol source was turned off, and another measurement of intensity was made. The ratio of these intensities is the true fractional transmission of the aerosol. Because the incident laser intensity was found to fluctuate in time, a second glass

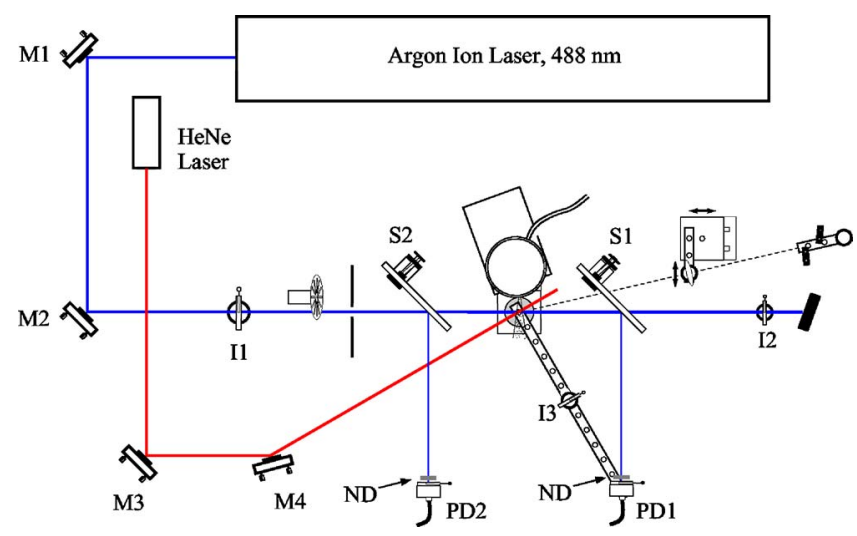

FIG. 5. The experimental setup used for measuring droplet density. This is similar to Fig. 2, however, here cover slides (S1 and S2) have been added to pick off a fraction of the laser beam before and after it interacts with the aerosol. A helium-neon laser was used for alignment purposes. From the measurements at PD1 and PD2 the aerosol density can be determined. ND refers to neutral density filters. pick-off slide was added before the aerosol. The signal recorded at PD1 was then normalized for laser intensity fluctuations by dividing by the signal measured at PD2. Finally, since the photodetectors are easily saturated, the laser intensity must be set to an appropriately low level. Although the pick-off slides only reflect a few percent of the beam to the photodetectors, the intensity may still be too high even for incident beam powers as low as $20 \mathrm{~mW}$. The problem of saturation was avoided by placing neutral density (ND) filters in front of the detectors. The ND filters used had an attenuation of $90 \%$.

In addition to measuring the distribution and density of droplets produced by the device, critical power measurements are also carried out. These measurements confirm that our device does indeed operate in the viscous regime of critical acceleration [see the discussion around Eqs. (5)-(7)], and can be used to estimate the power requirements of a device that will produce much smaller droplets. The critical power-the minimum power required to achieve atomization-is measured by monitoring the scatter of light near the surface of a driven fluid.

Unlike previous critical power studies, ${ }^{23}$ we cannot identify by eye the time at which critical power is reached; our droplets are too small to be seen individually by eye. We therefore rely on light scattering to detect the initial presence of droplets in our system. The apparatus used to make the critical power measurements is very similar to that shown in Fig. 2, except that only the reference photodetector (PD2) is needed in this case, and we use the helium-neon laser as the probe instead of the argon-ion laser. In these measurements, a baseline for the background signal is established by averaging the signal on PD2 over a $1 \mathrm{~s}$ interval with the laser turned on and the power to the piezo turned off. Once the baseline value for the background signal is established, the voltage on the dc supply that feeds the driver board is increased in increments of approximately $0.5 \mathrm{~V}$. At each increment the signal on PD2 is averaged over $1 \mathrm{~s}$ and recorded, both the ac voltage and ac current being fed directly to the piezo are measured, and the temperature of the fluid sample is taken. The ac voltage is measured using an oscilloscope and the ac current is measured using a high-frequency ammeter. The rms power delivered to the piezo is calculated from the product of these values, and represents an upper bound to the actual power delivered to the fluid. This procedure is repeated approximately 20 times. From these measurements we compare the power delivered to the piezo with the percentage of $1 \mathrm{~s}$ readings on the photodetector that rise above the background level. We assign the critical power to the point at which $10 \%$ of the readings rise above the background. We finish a data collecting run for a given fluid when multiple successive power readings result in $100 \%$ of the photodetector readings being above background-at this point, an aerosol visible to the eye is being produced. This procedure is repeated for each of the different fluids.

A fluid reservoir at a fixed height is used to fill and feed the fluid vessel that houses the piezo. This ensures that the fluid column height above the piezo remains constant throughout the measurements (at $3.3 \mathrm{~cm}$ ), and therefore that, for a given fluid, the mass is being driven is fixed. 


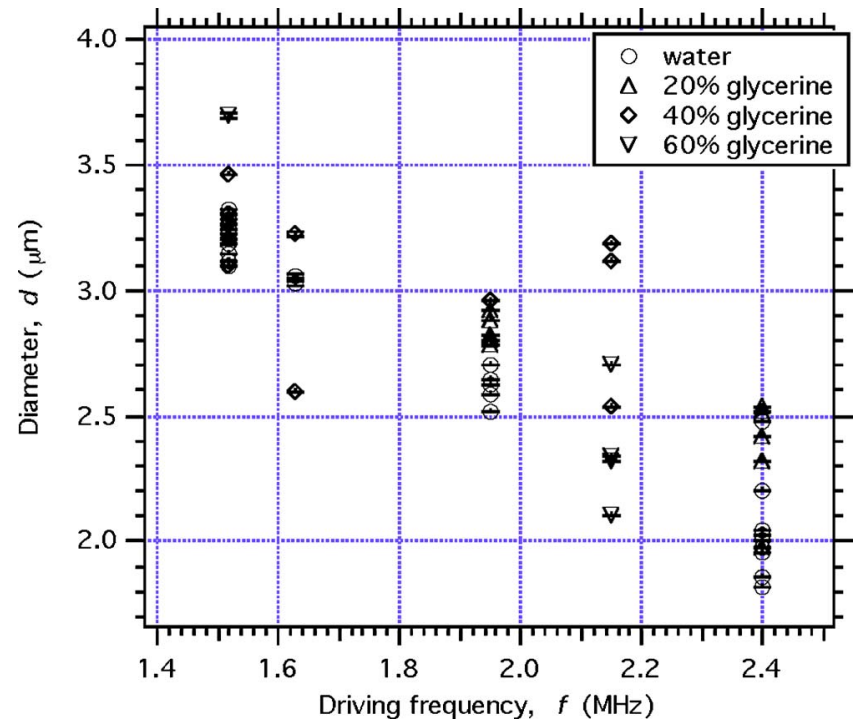

FIG. 6. The diameters of droplets that have been produced. The droplet diameters are shown as a function of ultrasonic driving frequency for water and water-glycerine mixtures. Variation in the droplet size for a particular fluid at a particular driving frequency is due to temperature differences in the fluid samples. The uncertainties in diameter represent the uncertainty in the peak diameter value of the lognormal distribution as determined by a chi-squared analysis.

\section{RESULTS}

The full results to date of droplet size measurements are shown in Fig. 6. In Fig. 7 these data are nondimensionalized using Eqs. (3) and (4) so that data from all of the fluids can be reduced to a single plot and easily compared with the theory of Faraday excitation. Data and the linear theory for capillary waves generated via Faraday excitation are shown to be in excellent agreement; the predicted power law is $-2 / 3$, and $-0.66 \pm 0.01$ is measured from the data. From these data, the constant of proportionality in Eq. (1) is measured to be $0.35 \pm 0.03$, in keeping with other literature values

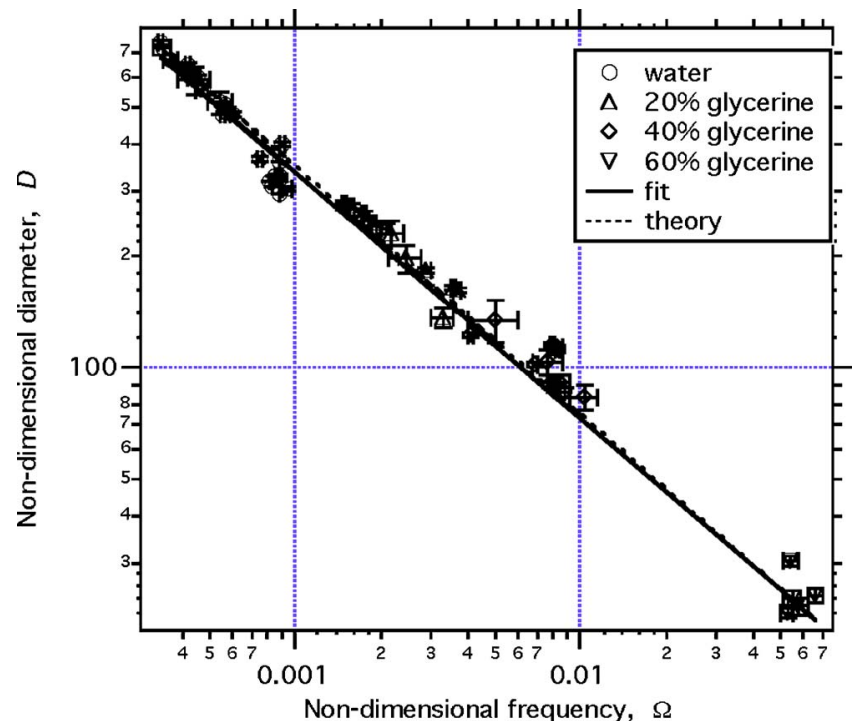

FIG. 7. Dimensionless diameter, D, vs dimensionless frequency, $\Omega$. The data match the inviscid theory predictions of droplet formation. The predicted power law is $-2 / 3$ [see Eqs. (1) and (3)], and $-0.66 \pm 0.01$ is measured from the data. of $0.34 .^{7}$ Together, Figs. 6 and 7 demonstrate that one can accurately predict the droplet sizes produced by ultrasonic atomization. This, in turn, means that by varying the driving frequency of the atomizer, or the fluid properties (density, surface tension, viscosity), droplets of a desired size can be produced. This is a significant advance for producing laser targets relevant to studies of fusion and nonlinear optics.

To this point, droplets distributions with a peak radius as small as $0.92 \pm 0.03 \mu \mathrm{m}$ have been produced, and the distributions typically have widths that are tens-of-percent of the peak value (see Figs. 3 and 4). It should be straightforward to make smaller droplets since the fluid dynamics predictions are well-known and have been verified with our apparatus. For instance, in the inviscid regime, droplet size, $d$, scales with driving frequency as $d \propto f^{-2 / 3}$. Thus, by increasing the ultrasonic driving frequency of the atomizer to $\sim 80 \mathrm{MHz}$ one can create droplets that are $\sim 0.1 \mu \mathrm{m}$ in radius. In this case, the droplets will have a size parameter near one and Mie enhancements will be important in experiments that study light-particle interactions. In practice, it may not be necessary to drive the fluids at such high frequencies because the droplet size can also be reduced in a controlled manner by decreasing the surface tension of the fluid through the use of surfactants.

In principle, it is a simple matter to make bigger droplets: one drives the fluid at a lower frequency. Lower frequency amplifiers and power supplies are cheaper and more common than their high frequency counterparts. For instance, extrapolating from the data shown in Fig. 7 using the inviscid scaling laws, water driven at $100 \mathrm{kHz}$ should produce droplets with $10 \mu \mathrm{m}$ radii. This should require less than $1 \%$ of the power used to drive fluids at megahertz frequencies. With a $10 \mu \mathrm{m}$ radius, the size parameter for our system would be $\sim 80$, meaning that these particles would act much like planar solids as far as Mie enhancements are concerned.

In addition to the distribution of droplet sizes, it is important to know the density of the aerosol being produced. We have measured the density of an aerosol produced when driving water at $2.42 \mathrm{MHz}$ using the setup shown in Fig. 5. The data shown in Fig. 8 were taken using this set up. From these measurements the density at the point of scatter is found to be $\sim 5 \times 10^{6}$ droplets $/ \mathrm{cm}^{3}$ or $\sim 10^{18}$ atoms $/ \mathrm{cm}^{3}$. This density compares well with the density of droplets produced using a gas jet, ${ }^{11,16}$ and to the deuterium cluster density used in fusion experiments. ${ }^{30}$

As described earlier, the critical power is determined by measuring the scatter of a helium-neon laser that is directed over the surface of a driven fluid. The results are shown in Fig. 9. We assign the value at which $10 \%$ of the readings gave a photodetector signal above the background as the critical power. This criterion is, of course, somewhat arbitrary, however similar results are achieved if one instead uses as the criterion the last power reading at $0 \%$, or the power reading that corresponds to $20 \%$. Figure 10 shows the results we obtain using a $10 \%$ scatter reading as our critical power criterion.

As expected, Fig. 10 shows that critical power is being measured in the viscous regime, as the power is dependent on viscosity and Eq. (5) shows that there is no viscosity 


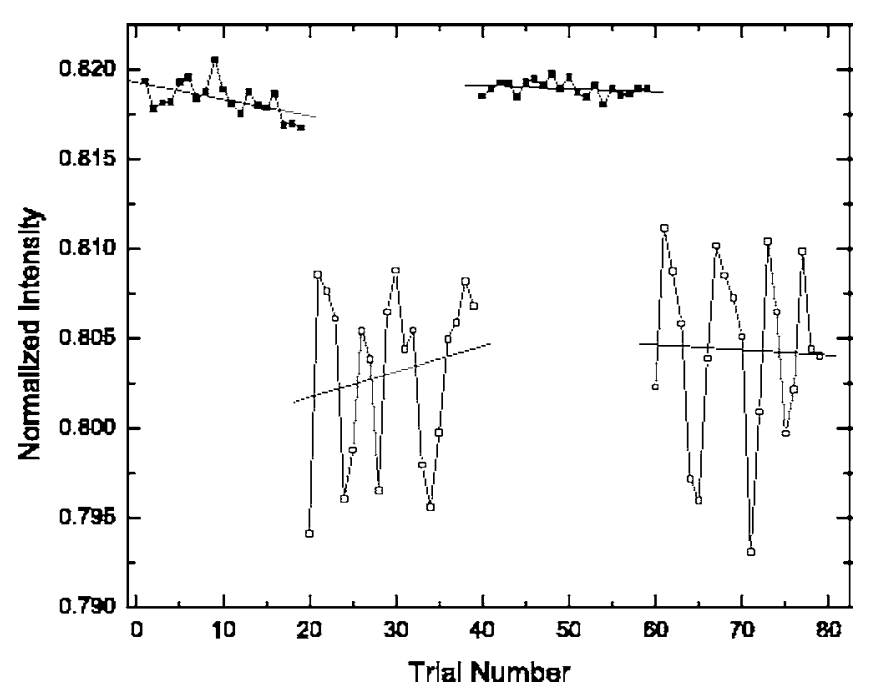

FIG. 8. Data taken for the droplet density measurement. Normalized data taken using the setup shown in Fig. 5 are shown. Closed squares represent data taken with no aerosol present, while the open squares show data taken with the aerosol source on. Attenuation of the laser beam due to the presence of the droplets is clear. The data were taken with a water sample driven at $2.42 \mathrm{MHz}$.

dependence in the inviscid regime. Further, the data indicate a linear dependence of critical power on fluid viscosity, consistent with Eq. (7). Equation (7) also predicts that the ratio of the slopes of the two fit-lines is the ratio of the driving frequencies squared: $(2.42 / 1.65)^{2}=2.15$, and Fig. 10 shows this ratio to be 2.0 for our $10 \%$ criterion.

Therefore, one can use Eq. (7) to extrapolate and determine the power requirements of a device that will produce droplets significantly smaller than $1 \mu \mathrm{m}$ droplets that will be useful in laser-driven fusion and nonlinear optics experiments. The deuterium-deuterium fusion cross section peaks at ion energies near $2 \mathrm{MeV}$. Assuming that Coulomb explo-

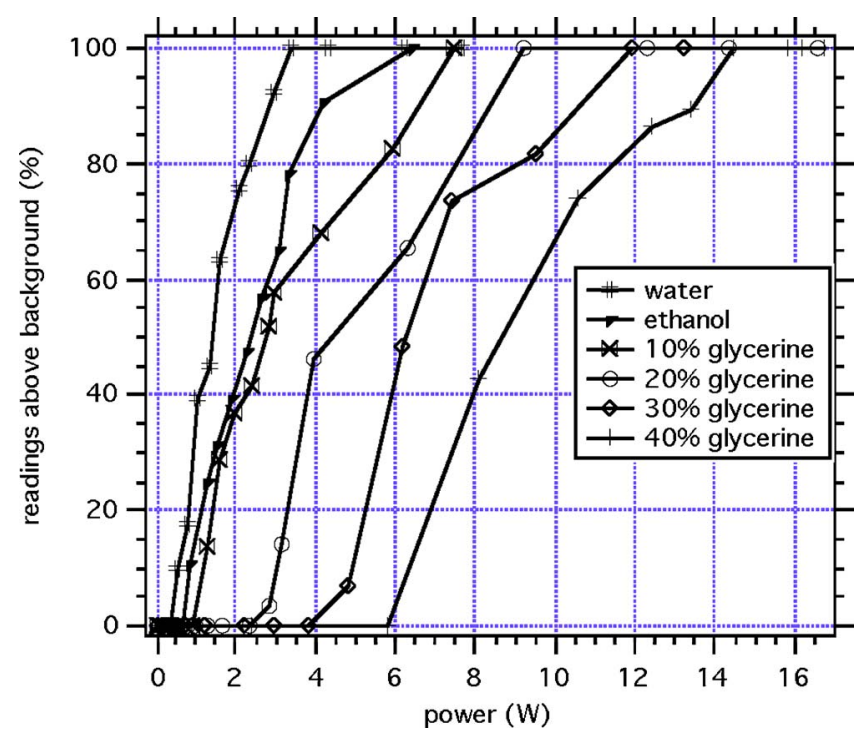

FIG. 9. The scatter of helium-neon laser light by the atomized fluid as a function of power delivered to the piezo. Data from six different fluid samples driven at $1.65 \mathrm{MHz}$ is shown. The scatter is quantified by the number of 1 s duration readings, out of approximately 20, that produced scatter signals above the background level. Critical power is estimated from these data as the power at which $10 \%$ of the readings are above background.

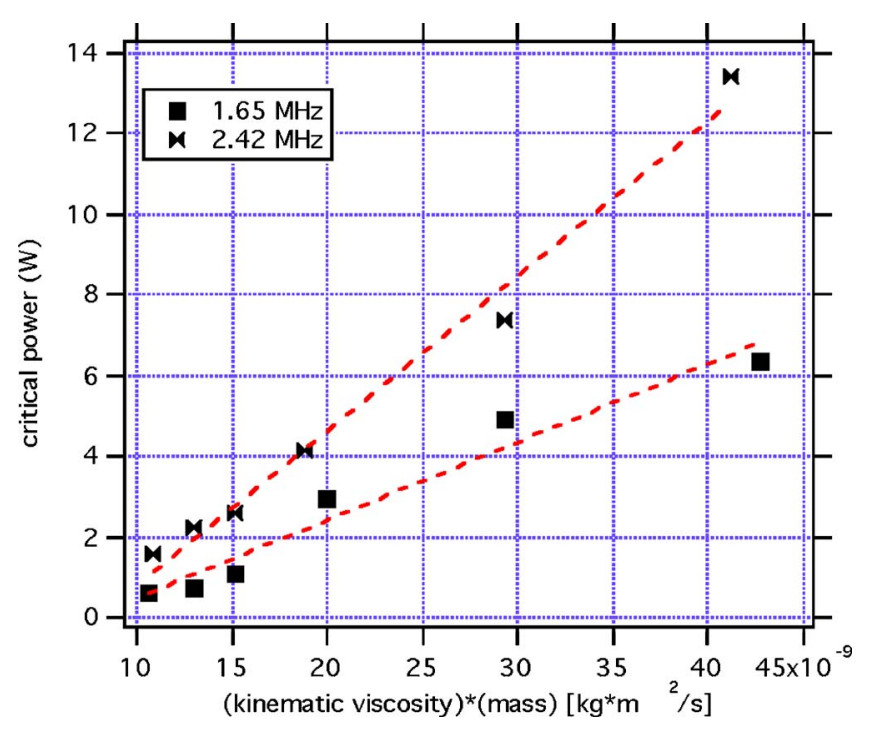

FIG. 10. Measurement of critical power as a function of fluid viscosity. Critical power is seen to scale linearly with the product of $m \nu$, as predicted by Eq. (7), and consistent with the scaling expected for a fluid driven in the viscous regime of critical acceleration. Data from six different fluid samples, driven at both 1.65 and $2.42 \mathrm{MHz}$, are shown. Linear fits are made to both sets of data $(1.65$ and $2.42 \mathrm{MHz})$, and the ratio of the slopes is found to be 2.0 , in keeping with the 2.15 ratio expected from Eq. (7).

sions dominate the energetics of the exploding clusters that produce these ions, it is optimal to have clusters, or heavy water droplets, of size $0.2 \mu \mathrm{m}$ or larger. Assuming that a droplet is irradiated with $800 \mathrm{~nm}$ light, from Eq. (11) it can also be seen that the size parameter reaches a value of 1 - the region where Mie enhancements are expected to be important-when water droplets have a size of $0.2 \mu \mathrm{m}$. Hence it is desirable to develop a source capable of producing droplets as small as $0.2 \mu \mathrm{m}$. Given the dependencies in Eq. (1), the most feasible way to generate such small droplets would be to drive the fluid at high frequency.

To create these small water droplets, it is advantageous to heat the fluid, as this will decrease the surface tension more significantly than it decreases density [see Tables I and II, and Eq. (1)]; it also has the advantage of reducing the viscosity and thereby decreasing input power requirements [see Table III and Eq. (7)]. Using surface tension and density values near $100{ }^{\circ} \mathrm{C}$, a $0.2 \mu \mathrm{m}$ water droplet would be created using a driving frequency of $85 \mathrm{MHz}$. The data shown in Fig. 10 verify that Eq. (7) can be used to determine the power requirements of a high-power amplifier running at this frequency. Assuming $10 \mathrm{~g}$ of water is driven (roughly the same as in the Fig. 10 data), the scaling from Eq. (7) shows that a multi-100 W amplifier can produce small droplets from the heated water.

We have presented the details of a robust device that is capable of producing micron-scale droplets through ultrasonic atomization. The droplet size produced by the device follows scaling laws predicted by fluid dynamics, making it straightforward to tailor droplets to the needs of a particular experiment. We have already used the device to test fluid theories in new frequency regimes, and look forward to building a higher-power, higher-frequency, secondgeneration device for use in nonlinear optics experiments and fusion research. 


\section{ACKNOWLEDGMENTS}

Part of this work was supported by the Beckman Research Fund at Harvey Mudd College. Acknowledgement is made to the donors of the Donors of the Petroleum Research Fund administered by the American Chemical Society for partial support of this research, for support by an award from the Research Corporation, and for the support of the National Science Foundation (No. PHY-0456898).

${ }^{1}$ J. Zweiback, T. E. Cowan, J. H. Hartley, R. Howell, K. B. Wharton, J. K. Crane, V. P. Yanovsky, G. Hays, R. A. Smith, and T. Ditmire, Phys. Plasmas 9, 3108 (2002).

${ }^{2}$ T. Ditmire, J. Zweiback, V. P. Yanovsky, T. E. Cowan, G. Hays, and K. B. Wharton, Nature (London) 398, 489 (1999).

${ }^{3}$ J. Zweiback, R. A. Smith, T. E. Cowan, G. Hays, K. B. Wharton,

V. P. Yanovsky, and T. Ditmire, Phys. Rev. Lett. 84, 2634 (2000).

${ }^{4}$ J. P. Dewitz, W. Hubner, and K. H. Bennemann, Z. Phys. D: At., Mol. Clusters 37, 75 (1996).

${ }^{5}$ N. Yang, W. E. Angerer, and A. G. Yodh, Phys. Rev. Lett. 87, 103902-1 (2001).

${ }^{6}$ J. I. Dadap, J. Shan, K. B. Eisenthal, and T. F. Heinz, Phys. Rev. Lett. 83, 4045 (1999).

${ }^{7}$ T. D. Donnelly, J. Hogan, A. Mugler, N. Schommer, M. Schubmehl, A. J. Bernoff, and B. Forrest, Phys. Fluids 16, 2843 (2004).

${ }^{8}$ T. D. Donnelly, M. Rust, I. Weiner, M. Allen, R. A. Smith, C. A. Steinke, S. Wilks, J. Zweiback, T. E. Cowan, and T. Ditmire, J. Phys. B 34, L313 (2001).

${ }^{9}$ N. Yang, W. E. Angerer, and A. G. Yodh, Phys. Rev. A 64, 045801 (2001).

${ }^{10}$ Y. Fukuda, K. Yamakawa, Y. Akahane, M. Aoyama, N. Inoue, H. Ueda, J. Abdallah, G. Csanak, A. Faenov, A. I. Magunov, T. A. Pikuz, I. Skobelev, A. S. Boldarev, and V. A. Gasilov, JETP Lett. 78, 115 (2003).
${ }^{11}$ L. C. Mountford, R. A. Smith, and M. H. R. Hutchinson, Rev. Sci. Instrum. 69, 3780 (1998).

${ }^{12}$ J. Kasparian, B. Kramer, J. P. Dewitz, S. Vajda, P. Rairoux, B. Vezin, V. Boutou, T. Leisner, W. Hubner, J. P. Wolf, L. Woste, and K. H. Bennemann, Phys. Rev. Lett. 78, 2952 (1997).

${ }^{13}$ J. W. G. Tisch, T. Ditmire, D. J. Fraser, N. Hay, M. B. Mason, E. Springate, J. P. Marangos, and M. H. R. Hutchinson, J. Phys. B 30, L709 (1997).

${ }^{14}$ T. D. Donnelly, T. Ditmire, K. Neuman, M. D. Perry, and R. W. Falcone, Phys. Rev. Lett. 76, 2472 (1996).

${ }^{15}$ F. Brunel, Phys. Rev. Lett. 59, 52 (1987).

${ }^{16}$ R. A. Smith, T. Ditmire, and J. W. G. Tisch, Rev. Sci. Instrum. 69, 3798 (1998).

${ }^{17}$ D. R. Symes, A. J. Comley, and R. A. Smith, Phys. Rev. Lett. 93, 145004-1 (2004).

${ }^{18}$ A. S. Boldarev, V. A. Gasilov, F. Blasco, C. Stenz, F. Dorchies, F. Salin, A. Faenov, T. A. Pikuz, A. I. Magunov, and I. Skobelev, JETP Lett. 73, 514 (2001).

${ }^{19}$ J. Miles and D. Henderson, Annu. Rev. Fluid Mech. 22, 143 (1990).

${ }^{20}$ T. B. Benjamin and F. Ursell, Proc. R. Soc. London, Ser. A 225, 505 (1954).

${ }^{21}$ K. Kumar and L. S. Tuckerman, J. Fluid Mech. 279, 49 (1994).

${ }^{22}$ C. Goodridge, L. A. Shi, W. Tao, and D. P. Lathrop, Phys. Rev. Lett. 76, 1824 (1996).

${ }^{23}$ C. L. Goodridge, W. T. Shi, H. G. E. Hentschel, and D. P. Lathrop, Phys. Rev. E 56, 472 (1997).

${ }^{24}$ D. Sindayihebura, M. Dobre, and L. Bolle, in ExHFT, 1997, Brussels.

${ }^{25}$ APC International, www.americanpiezo.com

${ }^{26}$ Thorlabs; www.thorlabs.com; part number PDA55

${ }^{27}$ I. Weiner, M. Rust, and T. D. Donnelly, Am. J. Phys. 69, 129 (2001).

${ }^{28}$ W. Wiscombe, Appl. Opt. 19, 1505 (1980).

${ }^{29}$ M. Kerker, The Scattering of Light and Other Electromagnetic Radiation (Academic, New York, 1969).

${ }^{30}$ T. Ditmire, J. Zweiback, V. P. Yanovsky, T. E. Cowan, G. Hays, and K. B. Wharton, Phys. Plasmas 7, 1993 (2000). 